\title{
A response-selection basis for the mixed-category, repeated-stimulus inferiority effect
}

\author{
ROBERT W. PROCTOR \\ Auburn University, Auburn, Alabama \\ and \\ GENE W. FOBER \\ U.S. Army Research Institute, Fort Benning Field Unit, Columbus, Georgia
}

\begin{abstract}
When subjects identify a target stimulus with an assigned keypress response, flanking noise stimuli produce interference if they signal an alternative response and slight facilitation if they are identical to the target. However, when the possible stimuli come from two distinct categories (letters and digits), interference also occurs if the noise letters are identical to the target. Four experiments were conducted to determine whether this mixed-category, repeated-stimulus inferiority effect is due to stimulus-identification or response-selection processes. The inferiority effect was (1) absent when letters were assigned to one response and digits to another; (2) absent when the target stimulus was named, rather than identified by a keypress response; and (3) eliminated when subjects practiced with mixed assignments of letters and digits. These findings converge on a response-selection basis for the inferiority effect.
\end{abstract}

When subjects are required to identify a target stimulus by a vocal naming response, a keypress response, or a lever-movement response, the response is slowed by the presence of extraneous noise stimuli that are assigned to a different response (the response-compatibility effect; e.g., B. A. Eriksen \& C. W. Eriksen, 1974; La Heij \& Vermeij, 1987; Proctor, 1981; Proctor \& Fober, 1985). When the noise stimuli indicate the same response as the target, reaction times are slightly faster when the stimuli are identical to the target than when they are not (B. A. Eriksen \& C. W. Eriksen, 1974; Proctor \& Fober, 1985; Taylor, 1977). We have referred to the latter finding as the repeated-stimulus superiority effect (Proctor \& Fober, 1985).

An exception to the repeated-stimulus superiority effect occurs when half of the stimuli assigned to each response are letters and half are digits. In this situation, responses are slower when the noise stimuli are identical to the target than when they are copies of a nonidentical stimulus, from the same category, that is assigned to the same response (Flowers \& Wilcox, 1982; LaBerge, 1981; Proctor \& Fober, 1985). This repeated-stimulus inferiority effect is of interest because the trials that are compared involve the same stimuli that produce the superiority effect when either only letters or only digits are used.

Previous explanations of repeated-stimulus inferiority in other tasks have attributed it to a stage of information

This research was supported in part by Grant AFOSR-88-0002 from the Air Force Office of Scientific Research to the first author. We would like to thank Lainie Dornier, Gil Reeve, Dan Weeks, Charles Eriksen, John Flowers, and Trammell Neill for helpful comments on earlier drafts. Requests for reprints should be sent to Robert W. Proctor, who is now at the Department of Psychological Sciences, Purdue University, West Lafayette, IN 47906. processing that sometimes is referred to as sensory preprocessing (e.g., Smith, 1968). These explanations have focused on sensory feature inhibition (e.g., Bjork \& Murray, 1977; Shapiro \& Krueger, 1983). For example, Bjork and Murray proposed a feature-specific inhibition model that predicts the inferiority for identical-noise trials because the target and noise are assumed to compete for access to the same set of feature analyzers. However, sensory feature inhibition cannot readily explain the inferiority effect obtained when the potential targets include both letters and digits, because the feature interactions for identical-noise trials should not depend on whether the stimuli are from one or two categories. Moreover, the evidence for sensory feature inhibition in other tasks has been questioned because of numerous procedural problems (C. W. Eriksen, Morris, Yeh, O'Hara, \& Durst, 1981; Estes, 1982; Santee \& Egeth, $1980,1982)$.

In addition to the sensory-preprocessing stage, models of visual information processing often include stages of stimulus identification and response selection (e.g., Smith, 1968). The locus for the repeated-stimulus inferiority effect could be at either of these processing stages. Previously, we noted three general types of possible explanations for the inferiority effect (Proctor \& Fober, 1985), two of which attribute the effect to stimulus-identification processes. First, because the members within the categories of letters and digits are more similar to each other than they are to members of the alternative category (Duncan, 1983; Krueger, 1984), the similarity distribution for the set of target-distractor combinations should be more heterogeneous when both letters and digits are used than when all stimuli are from only one category. Following logic similar to that used by Krueger (1978, 
1986) to explain the relation between same and different reaction times in matching tasks, the greater heterogeneity for the letter-digit stimulus set could slow the identification of the target stimulus for the identical-noise trials. Second, the repeated-stimulus inferiority effect could be due to feature interactions that occur in the identification processes, rather than in sensory preprocessing. For example, Estes (1982) proposed that criterion settings in identification processes are influenced by similarity relations between target and noise characters.

The third possible type of explanation for the repeatedstimulus inferiority effect attributes the effect to responseselection processes. That is, when a keypress response is required, not only must the target be identified, but the correct response must be selected. The mixing of letters and digits could cause subjects to adopt different responseselection strategies than they would otherwise. For example, response selection could proceed by deciding first whether the target was a letter or digit and then which specific response to make (Proctor \& Fober, 1985).

The primary purpose of the present study was to determine whether the repeated-stimulus inferiority effect has its basis in stimulus-identification or response-selection processes. Four experiments were conducted that followed the basic logic of manipulating variables that should affect response selection but not stimulus identification. All of the experiments included conditions in which responseselection requirements should be minimal. Experiment 1 examined a situation in which all of the letters were assigned to one keypress response and all of the digits to another, thus providing a simple response-selection rule. In Experiment 2, more direct, vocal naming responses were used. Experiments 3 and 4 examined performance in the basic keypress task after extended practice.

\section{EXPERIMENT 1}

Experiment 1 was designed to provide initial evidence about whether stimulus-identification or responseselection processes are responsible for the repeatedstimulus inferiority effect that occurs when two letters and two digits are assigned to each keypress response. In addition to this mixed assignment of letters and digits, an unmixed assignment was used in which four letters were assigned to one response and four digits to the other. The unmixed assignment alters and simplifies the responseselection process because it provides the following simple rule for responding (Flowers, 1985): Press one response key if the target is a letter and the other if the target is a digit. Thus, if the repeated-stimulus inferiority effect was obtained with the unmixed assignment, it would seem likely that the effect is based not on responseselection processes, but on stimulus-identification processes. If the inferiority effect was not obtained with the unmixed assignment, response-selection processes would be implicated. However, because the unmixed assignment potentially allows responding to be based on featural distinctions between letters and digits (e.g., Dun- can, 1983; Krueger, 1984), without the stimulus's being identified uniquely, the absence of the inferiority effect with this assignment would not completely rule out accounts based on stimulus identification.

Flowers (1985, Experiment 1) examined situations similar to those of the present experiment, in which some subjects used a mixed assignment of letters and digits and other subjects used an unmixed assignment. However, Flowers also varied stimulus onset asynchrony (SOA), rather than focusing on simultaneous presentation. Because the repeated-stimulus inferiority effect occurs only when the target and distractor are presented approximately simultaneously (Flowers \& Wilcox, 1982), the other SOAs are irrelevant for evaluating the effect. Flowers's mean data suggest that the repeated-stimulus inferiority effect was not present for the unmixed assignment of letters and digits when presentation was simultaneous, although the pertinent analyses were not reported. Our Experiment 1 was intended to provide more conclusive evidence.

\section{Method}

Apparatus and Stimuli. Stimuli were presented on the display screen of a Radio Shack TRS-80 Model 4 microcomputer. The stimuli were white on a dark background and were viewed at a distance of approximately $50 \mathrm{~cm}$. Responses were made by pressing either the $\mathrm{Z}$ or the ? key on the computer's keyboard with the left or right index finger, respectively. All timing was controlled by the computer, with reaction times recorded to the nearest millisecond.

The stimuli were uppercase letters and digits. Two sets of four letters (D, N, R, S and F, $L, T, X)$ and two sets of four digits (3, $4,5,7$ and 2, 6, 8, 9) were used. Each subject received one set of letters and one set of digits as target stimuli. For subjects who received a mixed assignment, two letters and two digits were assigned to the left response, and the other two letters and two digits to the right response. The specific assignments of stimuli to responses were counterbalanced across subjects. For subjects who received an unmixed assignment, the set of letters was assigned to one response and the set of digits to the other, with the assignments to the left and right responses counterbalanced across subjects.

Each display contained a target stimulus flanked by two noise stimuli, with each stimulus subtending a visual angle of approximately $.40^{\circ}$ in width and $.63^{\circ}$ in height as viewed by the subject. The target was displayed in a fixed location approximately $.68^{\circ}$ above an asterisk that served as a fixation spot, and the separation between the target and the noise stimuli was approximately $.40^{\circ}$. Each trial began with a 250 -msec offset of the fixation spot. Two hundred and fifty milliseconds after the fixation spot reappeared, the target and noise stimuli were presented simultaneously and remained in view until the subject responded. After a 1 -sec interval, the next trial sequence began.

Six conditions based on the relation between the target and noise were possible for the mixed assignment. These conditions will be illustrated using an example assignment of $R, S, 4,5$ to one response and $\mathrm{D}, \mathrm{N}, 3,7$ to the other. For the neutral-noise condition, the noise consisted of asterisks (e.g., ${ }^{*} R^{*} ;{ }^{*} 4^{*}$ ). For all other noise conditions, the noise stimuli were members of the target set. Identical noise consisted of stimuli that were the same as the target (e.g., RRR; 444); same-category/compatible noise consisted of the other member from the same category as the target that was assigned to the same response (e.g., SRS; 545); same-category/incompatible noise consisted of a member from the same category as the target that was assigned to the opposite response (e.g., DRD; 343); 
different-category/compatible noise consisted of a member from the category opposite to that of the target that was assigned to the same response (e.g., 4R4; R4R); and different-category/incompatible noise consisted of a member from the category opposite that of the target that was assigned to the opposite response (e.g., 3R3; D4D). For the unmixed assignment, the target-noise relations were the same, with the exception that the same-category/incompatible and different-category/compatible conditions were not applicable.

Lists of 144 trials were constructed that, when used with the mixed assignment, included 16 trials each from the neutral, identical, and same-category/compatible conditions and 32 trials each from the different-category/compatible, same-category/incompatible, and different-category/incompatible conditions. These proportions of trials were the same as those used by Flowers and Wilcox (1982) and Proctor and Fober (1985) and represent an equal number for all combinations of the specific target and noise stimuli. When the lists were used with the unmixed assignment, all 48 same-category trials now were compatible, whereas all 64 different-category trials were incompatible. Eight different lists were constructed, with the constraint that all possible target-noise combinations appeared equally often in both halves of each list in a pseudorandom order.

Subjects and Procedure. The subjects were 32 stidents at Auburn University who were enrolled in an introductory psychology course. Each subject participated in two sessions for extra credit toward his/her course grade. An additional 5 subjects, 4 from the mixedassignment condition and 1 from the unmixed-assignment condition, were omitted for exceeding either of two criteria in the test session: making errors on more than $10 \%$ of the trials or responding slower than $1 \mathrm{sec}$ on more than $10 \%$ of the trials.

The first session was a practice session intended to familiarize the subjects with the stimulus-response assignments. At the beginning of the practice session, each subject read written instructions that explained the task and assigned either two letters and two digits to each response (the mixed assignment) or four letters to one response and four digits to the other (the unmixed assignment). The instructions indicated that the target stimulus would occur in the location immediately above fixation, with irrelevant noise stimuli presented simultaneously in adjacent locations. The instructions also stressed that the subject should respond to the target as fast and accurately as possible. A block of 18 warm-up trials then was presented, followed by a set of 144 trials. The same procedure was followed in Session 2, which was the test session.

\section{Results}

Mean reaction times for correct responses and percentages of errors in the test session were obtained for each subject. Reaction times of less than 200 or more than $1,500 \mathrm{msec}$ (less than $1 \%$ of the responses) were excluded from the computations of mean reaction times. The summary means are shown in Table 1 . The results for the mixed assignment are considered first, followed by those for the unmixed assignment.

Mixed assignment. The results for the mixed assignment replicated previous findings. Analyses of all targetnoise conditions showed a significant difference in reaction times $[F(5,75)=4.86, p<.001]$ and error percentages $[F(5,75)=2.71, p<.03]$. More specific comparisons were performed between crucial conditions. First, the identical and same-category-compatible conditions were compared. Responses tended to be slower and less accurate for identical trials than for same-category/compatible trials [differences $(D s)=23 \mathrm{msec}$ and $1.9 \%$; $F \mathrm{~s}(1,15)=3.25$ and $3.15, p s<.10$ ]. Thus, although neither reaction times nor errors attained standard sig-
Table 1

Mean Reaction Times (RTs, in msec) and Percentages of Errors (\%Es) for Experiment 1 as a Function of Target-Noise Relation and Assignment

\begin{tabular}{cccccc}
\hline \multirow{2}{*}{$\begin{array}{c}\text { Target-Noise } \\
\text { Relation }\end{array}$} & \multicolumn{2}{c}{ Mixed Assignment } & & \multicolumn{2}{c}{ Unmixed Assignment } \\
\cline { 2 - 3 } \cline { 5 - 6 } & RT & \%E & & RT & \%E \\
\hline I & 597 & 3.1 & 550 & 3.0 \\
SC & 574 & 1.2 & 566 & 2.0 \\
SI & 597 & 5.9 & & \\
N & 563 & 3.1 & 562 & 1.4 \\
DC & 579 & 3.9 & & \\
DI & 603 & 3.9 & 595 & 3.9 \\
\hline
\end{tabular}

Note-I = identical; SC = same-category/compatible; $\mathrm{SI}=$ samecategory/incompatible; $\mathbf{N}=$ neutral; $\mathbf{D C}=$ different-category/compatible; $\mathrm{DI}=$ different-category/incompatible.

nificance levels, the patterns for both measures are consistent with the repeated-stimulus inferiority effect obtained previously by Flowers and Wilcox (1982), LaBerge (1981), and Proctor and Fober (1985).

Second, two-factor analyses of variance (category relation $\times$ response compatibility) were performed on the same-category/compatible, same-category/incompatible, different-category/compatible, and different-category/ incompatible conditions. Responses were slower $(D=$ $23 \mathrm{msec})$ and less accurate $(D=2.3 \%)$ when the response indicated by the noise was incompatible with the target response than when it was compatible $[F \mathrm{~s}(1,15)=25.4$ and $6.0, p s<.03]$. This outcome replicates the customarily obtained finding that incompatible noise interferes with responding (e.g., B. A. Eriksen \& C. W. Eriksen, 1974). The main effect of category relation was not significant for either reaction times or errors $(F \mathrm{~s}<1.0)$. The interaction of category relation with response compatibility also was not significant for reaction times $(F \mathbf{s}$ $<1.0)$, but was for errors $[F(1,15)=8.43, p<.02]$. The significant interaction indicates that the greater number of errors on incompatible trials was evident only for same-category noise (see Table 1).

Unmixed assignment. The unmixed assignment also showed reliable differences in reaction times between the respective target-noise conditions $[F(3,45)=5.34$, $p<.01]$, although the percentages of errors did not differ reliably $[F(3,45)=1.94, p>.10]$. Unlike the mixed assignment, responses tended to be faster on the identical trials than on the same-category/compatible trials $[F(1,15)$ $=1.47, p>.10$ ], although the error rate was slightly greater on the identical trials $(F<1.0)$. Most importantly, when mixed versus unmixed assignment was included as a factor, along with the identical versus samecategory/compatible distinction, the reaction times showed a significant condition $\times$ assignment interaction $[F(1,30)$ $=4.46, p<.05]$. This interaction indicates that reaction times were relatively faster for identical trials than for same-category/compatible trials when the assignment was unmixed.

The unmixed assignment did show an effect of target-noise compatibility, however, that was significant for the reaction times $[F(1,15)=6.67, p<.05]$ but not for 
the errors $[F(1,15)=3.75, p>.05]$. When mixed versus unmixed assignment was included as a factor, along with the distinction between the same-category/compatible and the different-category/incompatible conditions, neither reaction times nor the errors showed a condition $X$ assignment interaction $(F s<1.0)$. Thus, in contrast to the repeated-stimulus inferiority effect, the interference due to the incompatibility of the target and noise was independent of whether the assignment of letters and digits was mixed or unmixed.

\section{Discussion}

Experiment 1 closely replicated the basic phenomena obtained previously with a mixed assignment of letter and digit stimuli (Flowers \& Wilcox, 1982; LaBerge, 1981; Proctor \& Fober, 1985). Responses tended to be slower and less accurate when the noise was identical to the target than when it was nonidentical but response-compatible (the repeated-stimulus inferiority effect). Also, for nonidentical noise, responses were slower and less accurate when the noise was incompatible with the required response than when it was compatible (the responsecompatibility effect).

Use of an unmixed assignment had contrasting influences on these two phenomena. The repeated-stimulus inferiority effect was eliminated with the unmixed assignment, whereas the magnitude of the responsecompatibility effect was unaltered. That the compatibility effect was not altered by the unmixed assignment is consistent with the interpretation that the effect is a function of competing responses indicated by the noise stimuli (e.g., C. W. Eriksen \& B. A. Eriksen, 1979). That the repeated-stimulus inferiority effect was absent with the unmixed assignment shows clearly that the effect is not an automatic consequence of mixing letter and digit stimuli. This outcome is consistent with the hypothesis that the inferiority effect has its basis in the responseselection processes that are involved when the assignment of letters and digits is mixed. Alternatively, the effect could have its basis in stimulus-identification processes, but arise only when the stimuli must be identified uniquely for the assigned response to be made.

\section{EXPERIMENT 2}

The results of Experiment 1 suggest that the requirement of selecting the response assigned to the target stimulus may be responsible for the repeated-stimulus inferiority effect. Experiment 2 was designed to obtain additional evidence pertinent to this point by requiring vocal, naming responses, rather than keypress responses, to the alphanumeric stimuli. The basic logic of using naming responses is that they are highly overlearned; thus, the requirement of having to select the correct response to an identified stimulus is minimized (e.g., Forrin, Kumler, \& Morin, 1966; La Heij \& Vermeij, 1987). Therefore, if the repeated-stimulus inferiority effect is a function of response-selection processes, it should not occur when naming responses are required. Alternatively, because the naming task requires that the target stimulus be identified uniquely, the inferiority effect should occur if its locus is in stimulus-identification processes.

With naming responses, each alphanumeric stimulus has a unique response. As a consequence, there are no samecategory/compatible trials (or, for that matter, differentcategory/compatible trials) with which to compare the identical trials. However, the repeated-stimulus inferiority effect that occurs when letters and digits are mixed involves an increase in reaction time for the identical trials relative to all other types of trials (see Proctor \& Fober, 1985 , and also Table 1 of the present article). Thus, whether the inferiority effect occurs when letters and digits are mixed still can be determined in the present experiment.

\section{Method}

Forty-eight subjects from the same subject pool as in Experiment 1 participated for extra credit. None of the subjects had been in Experiment 1 or in any related experiments. Sixteen subjects were assigned randomly to each of three conditions. For the letters-only condition, all of the target and noise stimuli were letters, and for the digits-only condition, all of the stimuli were digits. For the letters-and-digits condition, both letter and digit stimuli were used. The lists of trials for the letters-and-digits condition were similar to those of Experiment 1, consisting of 18 practice trials and 144 test trials. The test trials included 16 identical trials, 16 neutral trials, 48 same-category/incompatible trials, and 64 different-category/ incompatible trials. For the letters-only and digits-only conditions, the test trials included 32 identical trials, 96 same-category/incompatible trials, and 16 neutral trials.

The primary change in procedure from Experiment 1 was that subjects were to name the target stimulus, rather than to make a keypress response. The vocal responses were registered by a GrasonStadler Model E7300A-1 voice-operated relay that was connected to an input port of the computer by a Lafayette interface system. The experimenter sat unobtrusively behind the subject, recording by hand the response for each trial. Immediately after completion of the session, the experimenter entered the trial numbers for which the responses were incorrect into the computer.

\section{Results}

Mean correct reaction times and proportions of errors in the test session were obtained as a function of condition and target-noise relation. The summary means for these data are shown in Table 2. As is apparent from the table, presentation condition had a negligible influence on the results. When only the identical, same-category/incompatible, and neutral relations common to all three presentation conditions were considered, there was neither a main effect of condition $(F \mathrm{~s}<1.0)$ nor an interaction with target-noise relation $[F \mathrm{~s}(4,90) \leq 1.59, p s>.15]$ on either reaction times or errors. Target-noise relation had a main effect for both measures $[F \mathrm{~s}(2,90) \geq 6.11$, $p s<.01]$, with responses being slower and less accurate for the incompatible trials than for the identical and neutral trials, and the latter two types of trials not differing reliably.

Thus, the naming task shows no evidence for a repeatedstimulus inferiority effect when letters and digits are mixed 
Table 2

Mean Reaction Times (RTs, in msec) and Percentages of Errors (\%Es) for Experiment 2 as a Function of Target-Noise Relation and Condition

\begin{tabular}{|c|c|c|c|c|c|c|}
\hline \multirow{2}{*}{$\begin{array}{c}\text { Target-Noise } \\
\text { Relation }\end{array}$} & \multicolumn{2}{|c|}{$\begin{array}{l}\text { Letters } \\
\text { Only }\end{array}$} & \multicolumn{2}{|c|}{$\begin{array}{l}\text { Digits } \\
\text { Only }\end{array}$} & \multicolumn{2}{|c|}{$\begin{array}{l}\text { Letters and } \\
\text { Digits }\end{array}$} \\
\hline & $\mathrm{RT}$ & $\overline{\phi E}$ & $\overline{\mathbf{R T}}$ & $\overline{\mathscr{F E}}$ & $\overline{\text { RT }}$ & $\overline{\% \mathrm{E}}$ \\
\hline I & 515 & 0.6 & 521 & 0.4 & 520 & 0.8 \\
\hline SI & 546 & 1.7 & 545 & 2.3 & 556 & 1.4 \\
\hline $\mathbf{N}$ & 521 & 2.4 & 520 & 0.4 & 531 & 1.2 \\
\hline DI & & & & & 559 & 1.4 \\
\hline
\end{tabular}

Note-I = identical; SI = same-category/incompatible; $\mathbf{N}=$ neutral; DI $=$ different-category/incompatible.

within a list of trials. This finding, along with the absence of the inferiority effect with the unmixed assignment in Experiment 1, suggests that the effect occurs only when subjects must select the appropriate response to make to a stimulus.

\section{EXPERIMENT 3}

When subjects perform a task with novel assignments of stimuli to responses, as when letters and digits are assigned to keypress responses, the mediating role of response-selection processes is regarded as considerable (e.g., Teichner \& Krebs, 1974). However, with practice, this role is reduced or eliminated (e.g., Proctor, Nunn, \& Pallos, 1983; Proctor \& Reeve, 1988; Schneider \& Fisk, 1983). Thus, if the repeated-stimulus inferiority effect is a consequence of response-selection processes, it should disappear as subjects become practiced with the task and assignments.

Because a test session consisting of 144 trials is brief, we decided to have subjects participate in two test sessions on each of 4 days. Each subject maintained the same stimulus-response assignment throughout the experiment. The prediction of the response-selection hypothesis is that the repeated-stimulus inferiority effect that is apparent on the initial day should be eliminated by the fourth day.

\section{Method}

Sixteen subjects from the same subject pool used for Experiments 1 and 2 (none of whom had participated in those experiments) participated for extra credit. Data from an additional 4 subjects were omitted, 2 for failing to complete the experiment, 1 for an experimenter error, and 1 for having an error rate that exceeded $10 \%$ in the first session. Each subject was tested on 4 days. The method was similar to that used for the mixed-assignment condition in Experiment 1 , except that only the letters $D, N, R$, and $S$ and the digits $3,4,5$, and 7 were used. Each subject was assigned two letters and two digits to each of the two index-finger responses. This assignment was maintained throughout the 4 days. On each day, subjects were tested on two sets of 18 practice and 144 test trials, with a 5-min break between sets.

\section{Results}

Mean correct reaction times and percentages of errors were obtained for each subject in each of the eight sets of trials. The means from the two sets on each day then were averaged to produce means for Sessions 1-4. These data are summarized in Table 3 as a function of targetnoise relation and session.

Session 1. Session 1 showed the major findings reported for the mixed-assignment condition in Experiment 1. Significant differences existed between the target-noise types for both reaction times $[F(5,75)=5.99, p<.001]$ and errors $[F(5,75)=3.32, p<.01]$. Follow-up analyses showed that responses were slower and less accurate on identical trials than on same-category/compatible trials (the repeated-stimulus inferiority effect) $[F \mathrm{~s}(1,31) \geq$ $12.5, p s<.01]$. This effect was stronger than that found in Experiment 1 because the first set of 144 trials was included in the data for the present experiment, but not for Experiment 1. For nonidentical trials, responses were slower and less accurate when the noise was incompatible than when it was compatible $[F \mathrm{~s}(1,15) \geq 22.7$, ps $<.001$ ]. Unlike Experiment 1, responses also were faster when the nonidentical noise was from the same category as the target $[F(1,15)=8.60, p<.02]$.

All sessions. An analysis of all sessions showed that reaction times decreased across sessions $[F(3,45)=27.3$, $p<.001$ ], whereas the percentage of errors did not $(F$ $<1.0)$. Both reaction times and errors showed significant effects of target-noise relation $[F \mathrm{~s}(5,75)=23.8$ and $12.8, p s<.001]$ as well as session $\times$ relation interactions $\left[F_{\mathrm{s}}(15,225)=2.53\right.$ and 2.13 , $\left.p \mathrm{~s}<.01\right]$. Although they were significant, the interactions were relatively weak because Sessions 2 and 3 yielded results that were intermediate but relatively similar to those of Session 4 . Therefore, it is most instructive to examine the results of Session 4 relative to those of Session 1 .

Unlike Session 1, Session 4 showed no repeatedstimulus inferiority effect. Responses on identical trials did not differ significantly from those on samecategory/compatible trials $(F \mathrm{~s}<1.0)$; they were $2 \mathrm{msec}$ slower and $0.8 \%$ more accurate. However, the responsecompatibility effect was still present for both reaction times and errors $\left[F_{\mathrm{s}}(1,15)=67.0\right.$ and $\left.39.9, p \mathrm{~s}<.001\right]$, with a magnitude only slightly less than that in the first session. Responses also still were faster when the target and noise were from the same category than when they were not $[F(1,15)=7.26, p<.02]$.

Thus, Experiment 3 showed a dissociation between the inferiority and compatibility effects similar to the dissoci-

Table 3

Mean Reaction Times (RTs, in msec) and Percentages of Errors (\%Es) in Experiment 3 as a Function of Condition and Session

\begin{tabular}{|c|c|c|c|c|c|c|c|c|}
\hline \multirow{2}{*}{$\begin{array}{c}\text { Target-Noise } \\
\text { Relation }\end{array}$} & \multicolumn{2}{|c|}{ Session 1} & \multicolumn{2}{|c|}{ Session 2} & \multicolumn{2}{|c|}{ Session 3} & \multicolumn{2}{|c|}{ Session 4} \\
\hline & RT & $\% \mathrm{E}$ & RT & $\% \mathrm{E}$ & RT & $\% \mathrm{E}$ & RT & $\% \mathrm{E}$ \\
\hline I & 600 & 6.9 & 493 & 7.0 & 468 & 7.9 & 446 & 5.3 \\
\hline SC & 560 & 5.8 & 484 & 5.2 & 461 & 6.5 & 444 & 6.1 \\
\hline SI & 591 & 4.8 & 510 & 8.1 & 494 & 9.0 & 468 & 9.8 \\
\hline $\mathbf{N}$ & 580 & 4.6 & 492 & 7.5 & 466 & 7.6 & 449 & 6.2 \\
\hline DC & 589 & 4.2 & 496 & 9.4 & 463 & 5.9 & 451 & 5.7 \\
\hline DI & 600 & 5.4 & 517 & 10.6 & 488 & 7.9 & 471 & 9.4 \\
\hline
\end{tabular}

Note-I = identical; SC = same-category/compatible; SI = samecategory/incompatible; $\mathbf{N}=$ neutral; $\mathbf{D C}=$ different-category/compatible; DI = different-category/incompatible. 
ation shown in Experiment 1. This dissociation is important because it clearly indicates that performance on the identical trials changed more than did performance in the other target-noise conditions. The decrease in reaction time for the identical-noise trials from the first session to the fourth session was $154 \mathrm{msec}$, whereas the decrease was $127 \mathrm{msec}$ for the response-compatible trials and $124 \mathrm{msec}$ for the response-incompatible trials.

\section{Discussion}

The primary finding of Experiment 3 was that the repeated-stimulus inferiority effect was eliminated with practice. Whereas in Session 1 the reaction times for identical trials were $40 \mathrm{msec}$ slower than those for samecategory/compatible trials, in Session 4 they were only $2 \mathrm{msec}$ slower. The elimination of the inferiority effect was due to the identical condition's changing relative to all of the other target-noise conditions. That the repeatedstimulus inferiority effect disappeared with practice is consistent with the hypothesis that the effect is a consequence of response-selection processes.

In two previous experiments (Flowers \& Wilcox, 1982, Experiment 3; Proctor \& Fober, 1985, Experiment 1), subjects were given extended practice with mixed assignments of letters and digits, and neither one showed a consistent change in the magnitude of the repeated-stimulus inferiority effect. These earlier experiments differed from the present one, however, in that the nature of the task was varied between blocks of trials. Proctor and Fober tested subjects in four sessions, one per day, with each subject receiving an initial practice session with a mixed list of letters and digits. Three test sessions were then administered, counterbalanced for order, that involved letters only, digits only, and mixed presentation of letters and digits. Although not reported in the article, the size of the inferiority effect was roughly comparable whether subjects received the mixed list on Day 2, Day 3, or Day 4. However, only 8 subjects were tested with each specific order of conditions, and the extra "practice" for subjects who were tested with the mixed list on Day 4 was with only letters on one day and only digits on the other. This procedure may not be effective for learning the entire set.

Flowers and Wilcox (1982) tested subjects in seven sessions, in all of which both letters and digits were used. However, the onset between the target and distractors was varied between blocks, as was the target-distractor spacing. Simultaneous onset of the target and distractors, which is the situation that yields the repeated-stimulus inferiority effect, occurred on only one-sixth of the trials. Flowers and Wilcox reported that an analysis of all conditions showed no interaction with sessions. However, because they did not analyze the simultaneous-onset trials separately, it is unclear whether the repeated-stimulus inferiority effect decreased with practice. Assuming that it did not, the implication of their findings is that the benefit of practice may be due not to learning the stimulus- response assignments, but to experience with the specific conditions that produce the inferiority effect.

\section{EXPERIMENT 4}

Experiment 4 was designed to determine whether acquisition of the stimulus-response assignment alone is sufficient to eliminate the repeated-stimulus inferiority effect. Subjects were tested for four sessions, as in Experiment 3. However, for the first three sessions, only a single target stimulus, with no noise, was presented on each trial. Adjacent noise letters were introduced in Session 4. If practice with the stimulus-response assignment is sufficient to eliminate the repeated-stimulus inferiority effect, the results for Session 4 should resemble those obtained for Session 4 in Experiment 3; that is, no inferiority effect should be apparent. In contrast, if experience with the specific noise conditions is necessary, the inferiority effect should be obtained in Session 4 .

\section{Method}

Sixteen subjects from the same subject pool used for Experiments 1-3 (none of whom had participated in the earlier experiments) were tested for four sessions, as in Experiment 3. The only change in method from that experiment was that the computer program used for Sessions 1-3 was modified to present only the target stimulus on each trial.

\section{Results and Discussion}

Sessions 1-3. Mean reaction times decreased over Sessions 1-3 [567, 501, and $492 \mathrm{msec}$ for Sessions 1, 2, and 3 , respectively; $F(2,30)=13.8, p<.001]$. The percentages of errors did not differ reliably $[3.6 \%, 3.2 \%$, and $5.4 \%$ for Sessions 1, 2, and 3, respectively; $F(2,32)=$ $2.26, p>.10$ ]. The inflated error rate for Session 3 was due primarily to one subject.

Session 4. The results for the crucial fourth session were similar to those for the fourth session in Experiment 3. Both reaction times and errors differed reliably across the six conditions $[F \mathrm{~s}(5,75)=10.6$ and $2.87, p s<.05$; see Table 4]. However, the identical and same-category/compatible conditions did not differ significantly on either measure $(F \mathrm{~s}<1.0)$, indicating that the repeated-stimulus

Table 4

Mean Reaction Times (RTs, in msec) and Percentages of Errors (\%Es) for Session 4 of Experiment 4, as a Function of Target-Noise Relation

\begin{tabular}{ccc}
\hline $\begin{array}{c}\text { Target-Noise } \\
\text { Relation }\end{array}$ & RT & \%E \\
\hline I & 527 & 3.5 \\
SC & 524 & 3.0 \\
SI & 567 & 5.3 \\
N & 529 & 3.2 \\
DC & 539 & 3.3 \\
DI & 565 & 5.7 \\
\hline
\end{tabular}

Note-I = identical; SC = same-category/compatible; SI = samecategory/incompatible; $N=$ neutral; $\mathbf{D C}=$ different-category/compatible; $\mathrm{DI}=$ different-category/incompatible. 
inferiority effect was absent. The two-factor analysis of category relation (same, different) and response compatibility (compatible, incompatible) showed that, as in the previous experiments, responses were slower and less accurate for incompatible trials than for compatible trials $[F \mathrm{~s}(1,15) \geq 18.9, p s<.001]$. Neither the main effect of category relation nor its interaction with response compatibility was significant $(F \mathrm{~s} \leq 1.41, p \mathrm{~s}>.25)$.

Thus, the results of Experiment 4 are straightforward. Practice with the stimulus-response assignments alone is sufficient to eliminate the repeated-stimulus inferiority effect.

\section{GENERAL DISCUSSION}

When subjects identify a target stimulus that is flanked by noise stimuli, responses usually are slightly faster and more accurate when the noise is identical to the target than when it is a different stimulus assigned to the same response (B. A. Eriksen \& C. W. Eriksen, 1974; C. W. Eriksen \& B. A. Eriksen, 1979; Taylor, 1977). In contrast, when the stimulus set is composed of both letters and digits, responses are slower and less accurate when the noise is identical to the target (Flowers \& Wilcox, 1982; LaBerge, 1981; Proctor \& Fober, 1985). The present experiments again confirmed the repeated-stimulus inferiority effect that is obtained when the stimulus set is composed of letters and digits.

In terms of information-processing models, the repeated-stimulus inferiority effect could be a function of either sensory-preprocessing, stimulus-identification, or response-selection processes. That the inferiority effect occurs only when the stimulus set consists of both letters and digits argues against a sensory-preprocessing basis. That is, because sensory interactions usually are assumed to be an automatic consequence of the stimuli that are presented (e.g., Bjork \& Murray, 1977), the repeatedstimulus inferiority effect would be expected also to occur when all stimuli are from the same category.

The present experiments were designed to distinguish between stimulus-identification and response-selection explanations for the repeated-stimulus inferiority effect. The results from four experiments converged on a responseselection basis. Experiment 1 demonstrated that the inferiority effect did not occur when the letters were assigned to one response and the digits to another. The major difference between this situation and the situation in which both letters and digits are assigned to each response is that response-selection requirements are less in the former situation. Thus, the absence of the repeated-stimulus inferiority effect in that situation suggests that the effect may be a function of the more complex response-selection processes that are required when the assignments of letters and digits to responses are mixed.

Experiments 2, 3, and 4 provided further evidence that the repeated-stimulus inferiority effect is a function of response-selection processes. Experiment 2 showed that mixing letters and digits does not produce a repeated- stimulus inferiority effect when a vocal naming response, rather than a keypress response, is made to the target. One characteristic of the naming response is that it is highly overlearned; thus, response-selection requirements are minimized. Experiment 3 demonstrated that the inferiority effect obtained when both letters and digits are assigned to each of two keypress responses disappears with practice. Moreover, Experiment 4 showed that practice with responding to targets alone was sufficient to eliminate the repeated-stimulus inferiority effect when subjects subsequently were tested with noise stimuli present. Again, one of the major changes that occurs with practice is that the role of response-selection processes decreases (Teichner \& Krebs, 1974).

Although the results of the present experiments converge on a response-selection basis for the mixedcategory, repeated-stimulus inferiority effect, they do not provide a specific characterization of the processes that underlie the effect. One possibility is that the use of two distinct categories encourages subjects to adopt a hierarchical selection process in which the target is first classified as a letter or digit and then the appropriate response is selected. To date, however, we have not been able to determine why a hierarchical selection process would selectively introduce difficulties for identical trials only.

An alternative possibility relates the repeated-stimulus inferiority effect more closely to the specific requirements of the target-distractor task. In a typical choice-reaction task, only a single stimulus is presented on each trial, with that stimulus being the target to which the subject must respond (e.g., Proctor \& Reeve, 1985). However, in the target-distractor task, the target stimulus is flanked by noise stimuli that may signal an incorrect response. Thus, whereas stimulus identity alone is sufficient for selecting a response in the typical choice-reaction task, it is not in the target-distractor task. Accurate responding in the latter task also requires determination of the relative locations in which the specific stimuli occurred.

This requirement of determining which stimulus is in the target location apparently underlies the responsecompatibility effect (see, e.g., C. W. Eriksen \& Schultz, 1979). Most importantly, as the spacing between the target and noise locations is increased, which should reduce confusion about relative location, the responsecompatibility effect decreases substantially in magnitude (B. A. Eriksen \& C. W. Eriksen, 1974; C. W. Eriksen \& B. A. Eriksen, 1979). Consistent with evidence that stimulus identification can occur without correct spatial localization (e.g., Estes, Allmeyer, \& Reder, 1976), C. W. Eriksen and Schultz's continuous-flow account of the response-compatibility effect assumes explicitly that identity and location are processed at least partly independently. According to this account, activation of both the correct and incorrect responses occurs on responseincompatible trials until the locations of the respective stimuli are determined. The competition produced by activation of the two responses is the source of the responsecompatibility effect. 
When the target and noise are identical, a response conceivably could be made without location being determined, because only a single stimulus identity (and corresponding response) is indicated by the target and noise. However, if uncertainty were to exist regarding whether a second stimulus identity was present, the response could not be made until this uncertainty was resolved. Thus, the repeated-stimulus inferiority effect may arise from uncertainty about the presence of a second stimulus identity. Such uncertainty may occur when letters and digits are mixed if the two stimulus categories are partitioned, with letters and digits identified by separate subsystems (e.g., Corcoran \& Jackson, 1977). In other words, if the subsystem for identifying letters indicates only a single identity, response selection may be delayed pending a possible identification from the subsystem for digits, and vice versa.

One interesting aspect of the present experiments was the dissociation of the repeated-stimulus inferiority and response-compatibility effects. The inferiority effect was eliminated by the use of an unmixed assignment of letters and digits to keypress responses, by the use of vocal naming responses, and by practice with a mixed assignment of letters and digits to keypress responses. However, the response-compatibility effect was relatively uninfluenced by these manipulations. This lack of influence on the response-compatibility effect is not surprising, because, regardless of the specific response conditions, accurate responding on response-incompatible trials requires that the relative locations of the target and noise be resolved.

In contrast, the repeated-stimulus inferiority effect should occur only in situations in which uncertainty exists on identical trials about whether a second stimulus identity is present. The absence of the inferiority effect when the role of response selection is minimized therefore suggests that uncertainty exists only when there are not relatively direct links between stimuli and responses. Response selection can be characterized as involving the translation between stimulus and response codes, with this translation based on declarative representations of the task requirements (Anderson, 1983; Proctor \& Reeve, 1988). With practice, automatic task-specific procedures are acquired that produce specific outputs (overt or covert responses) when the defining conditions occur. When performance of the target-distractor task relies on automatic procedures that have been acquired either previously (i.e., the category distinction, Experiment 1 ; the naming response, Experiment 2) or in the experiment (i.e., with practice in Experiments 3 and 4), the production of a single response by the automatic procedures apparently provides a sufficient basis for responding.

The preceding account of the repeated-stimulus inferiority effect, although speculative, makes an important general point. In complex tasks, such as the one examined in the present study, the information on which responses are based may vary across conditions. Detailed analyses of the task requirements are necessary to obtain a com- plete understanding of the underlying psychological processes.

\section{REFERENCES}

Anderson, J. R. (1983). The architecture of cognition. Cambridge. MA: Harvard.

Bjork, E. L., MURray, J. T. (1977). On the nature of input channels in visual processing. Psychological Review, 84, 472-484.

Corcoran, D. W. J., JACKSON, A. (1977). Basic processes and strategies in visual search. In S. Dornic (Ed.), Attention and performance VI (pp. 387-411). Hillsdale, NJ: Erlbaum.

Duncan, J. (1983). Category effects in visual search: A failure to replicate the "oh-zero" phenomenon. Perception \& Psychophysics, 34, 221-232.

Eriksen, B. A., \& Eriksen, C. W. (1974). Effects of noise letters upon the identification of a target letter in a nonsearch task. Perception \& Psychophysics, 16, 143-149.

Eriksen, C. W., Eruksen, B. A. (1979). Target redundancy in visual search: Do repetitions of the target within the display impair processing? Perception \& Psychophysics, 26, 195-205.

Eriksen, C. W., Morris, N., Yeh, Y.-Y., O'Hara, W., Durst, R. T. (1981). Is recognition accuracy really impaired when the target is repeated in the display? Perception \& Psychophysics, 30 , 375-385.

ERIKSEN, C. W., Schultz, D. W. (1979). Information processing in visual search: A continuous flow conception and experimental results. Perception \& Psychophysics, 25, 249-263.

EsTES, W. K. (1982). Similarity-related channel interactions in visual processing. Journal of Experimental Psychology: Human Perception \& Performance, 8, 353-382.

Estes, W. K., All.meyer, D. H., \& Reder, S. M. (1976). Serial position functions for letter identification at brief and extended exposure durations. Perception \& Psychophysics, 19, 1-15.

Flowers, J. H. (1985). Priming effects in perceptual classificarion. Unpublished manuscript.

FLowers, J. H., Wilcox, N. (1982). The effect of flanking context on visual classification: The joint contribution of interactions at different processing levels. Perception \& Psychophysics, 32, 581-591.

Forrin, B., Kumler, M. L., Morin, R. E. (1966). The effects of response code and signal probability in a numeral-naming task. Canadian Joumal of Psychology, 20, 115-124.

Krueger, L. E. (1978). A theory of perceptual matching. Psychological Review, 85, 278-304.

KRUEGer, L. E. (1984). The category effect in visual search depends on physical rather than conceptual differences. Perception \& Psychophysics, 35, 558-564.

KRUEGer, L. E. (1986). Positive effect of heterogeneity of difference on the same-different disparity in letter matching. Perception \& Psychophysics, 39, 117-122.

LABERGE, D. (1981). Unitization and automaticity in perception. In J. $\mathbf{H}$. Flowers (Ed.), 1980 Nebraska Symposium on Motivation: Cognitive processes (Vol. 28, pp. 53-71). Lincoln: University of Nebraska Press.

LA HeIJ, W., VermeIJ, M. (1987). Reading versus naming: The effect of target set size on contextual interference and facilitation. Perception \& Psychophysics, 41, 355-366.

Proctor, R. W. (1981). A unified theory for matching-task phenomena. Psychological Review, 88, 291-326.

Proctor, R. W., \& Fober, G. W. (1985). Repeated-stimulus superiority and inferiority effects in the identification of letters and digits. Perception \& Psychophysics, 38, 125-134.

Proctor, R. W., Nunn, M. B., Pallos, I. (1983). The influence of metacontrast masking on detection and spatial-choice judgments: An apparent distinction between automatic and attentive response mechanisms. Joumal of Experimental Psychology: Human Perception \& Performance, 9, 278-287.

Proctor, R. W., Reeve, T. G. (1985). Compatibility effects in the assignment of symbolic stimuli to discrete finger responses. Joumal of Experimental Psychology: Human Perception \& Performance, 11, 623-639. 
Proctor, R. W., \& Regve, T. G. (1988). The acquisition of task-specific productions and modification of declarative representations in spatial precuing tasks. Joumal of Experimental Psychology: General, 117, 182-196.

SANTEE, J. L., \& EGETH, H. E. (1980). Interference in letter identification: A test of feature-specific inhibition. Perception \& Psychophysics, 27, 321-330.

SANTEe, J. L., EGETH, H. E. (1982). Do reaction time and accuracy measure the same aspects of letter recognition? Joumal of Experimental Psychology: Human Perception \& Performance, 8, 489-501.

SCHNEIDER, W., \& Fisk, A. D. (1983). Attention theory and mechanisms for skilled performance. In R. A. Magill (Ed.), Memory and control of action (pp. 119-143). Amsterdam: North-Holland.
SHAPiro, R. G., KRUEGER, L. E. (1983). Effect of similarity of surround on target-letter processing. Journal of Experimental Psychology: Human Perception \& Performance, 9, 547-559.

SMITH, E. E. (1968). Choice reaction time: An analysis of the major theoretical positions. Psychological Bulletin, 69, 77-100.

TAYLOR, D. A. (1977). Time course of context effects. Journal of Experimental Psychology: General, 106, 404-426.

Teichner, W. H., Krebs, M. J. (1974). Laws of visual choice reaction time. Psychological Review, 81, 75-98.

(Manuscript received November 5, 1987; revision accepted for publication February 12, 1988.) 